\title{
98. Cranioplasty Using Bone treated with Ethylendiamine
}

\author{
Miyoshi Urabe, Takashi Tsubokawa, Makoto Kikuchi, \\ Moriro Miyanaga, Nagamitsu Mukai and Haruhide Ito \\ Department of Surgery, School of Medicine, University of Kanazawa
}

Bone grafts of homologous and heterologous (dog) origin which were treated with ethylendiamine were implanted into traumatic or inflammatory defect of human skulls in 8 cases. The size of the grafts ranged from the circular one with a diameter of $2 \mathrm{~cm}$ to a dimension of 7 by $8 \mathrm{~cm}$. These cases were followed up for one year. Histological examination was performed on a case who had an implantation of both homologous and heterogous bones.

The results showed that the ethylendiamine-treated bones were revascularized, remodelled and incorporated progressively with tissue, so that solid union of the graft to host was accomplished. Ethylendiamine-treated bone was an appropriate graft regardless of the source.

\section{Severe Mental Disorder following Head Injury}

\author{
Toshisuke Yamamoto, Akio Yamagiwa, Yoichi Yoshida \\ and Tsuyoshi Yamashita \\ Department of Surgery, Mie Prefectural University School of Medicine
}

Hisashi HATTORI

Department of Neuro-Psychiatry, Mie Prefectural University School of Medicine

In recent years, there are increasing numbers of head injury. Most cases of them are able to live usual social lifes, however not a few cases were impossible to live usual lifes due to severe mental disorder, in spite of long term treatment.

We experienced six patients who had prolonged coma and became to "Decerebration" ("Apallisches Syndrom") following severe head injury: four cases of them were irreversible, and two cases were survived but developed severe mental disorder.

In these cases, typical or atypical decerebration developed, and then amnestic syndrom; intense retrograde amnesia and remarkable posttraumatic amnesia with disorder of impressibility after prolonged coma. 